\title{
Antiplatelet Therapy for Elderly Patients: When Less May Be More
}

\author{
Roberto Esporcatte ${ }^{1,2}$ and Fernando Oswaldo Dias Rangel ${ }^{1(1)}$ \\ Hospital Pró-Cardíaco, ${ }^{\prime}$ Rio de Janeiro, RJ - Brazil \\ Faculdade de Ciências Médicas - Hospital Universitário Pedro Ernesto (UERJ), ${ }^{2}$ Rio de Janeiro, $R J$ - Brazil
}

Editorial related to the article: Is There Safety in the Use of Clopidogrel Loading Dose in Patients Over 75 Years of Age with Acute Coronary Syndrome?

"Seventy is old enough. After that there is too much risk"

Mark Twain, 1897

In the last two decades, double antiplatelet therapy, together with percutaneous coronary intervention (PCI), has become a cornerstone of acute coronary syndrome (ACS) treatment and its use is recommended as early as possible, although there are many unanswered questions about the efficacy and safety of loading dose (LD), best moment of administration, clinical scenarios and, especially, its use in elderly patients. Despite the fact that elderly patients represent approximately $30 \%$ of the population admitted for ACS, they are virtually excluded from large clinical trials, receive fewer diagnostic and therapeutic approaches and have more ischemic and hemorrhagic complications. ${ }^{1}$

Pretreatment and LD of P2Y12 antagonists bring potential benefits of increased myocardial infarctionrelated patency rate and reduction of peri-procedure myocardial infarction, stent thrombosis and vascular reocclusion. In contrast, there is a higher risk of bleeding related to PCI and possible myocardial revascularization surgery and inadvertent use in patients with contraindications.

The study published in this issue, by Soeiro et al., ${ }^{2}$ brings important questions regarding the safety of clopidogrel LD for elderly patients admitted for ACS. Clinical outcomes were analyzed in a sample of patients

\section{Keywords}

Platelet Aggregation Inhibitors/therapeutic use; Aged; Hemorrhage; Acute Coronary Syndrome. with mean age of 80 years, according to a LD of $75 \mathrm{mg}$ (group I, $\mathrm{n}=129$ ) or 300 to $600 \mathrm{mg}$ (group II, $\mathrm{n}=45$ ).

As a result of the retrospective, observational, unicentric trial design and a small number of patients, there was great heterogeneity between groups I and II regarding important risk factors like diabetes mellitus, arterial hypertension, dyslipidemia and ST-segment elevation myocardial infarction (STEMI). Likewise, there is also a relevant difference regarding the use of myocardial reperfusion strategies (including PCI, fibrinolysis or myocardial revascularization surgery).

This way, group I apparently had higher ischemic risk, but received fewer myocardial reperfusion therapies. This may also compromise the comparative analysis of major clinical outcomes, with equality in the total number of combined adverse events but numerical differences in isolated outcomes, with higher rates of reinfarction, shock and mortality in group I and more hemorrhagic complications and ischemic stroke in group II. Nevertheless, hemorrhage rate was significant higher in group II: $8.5 \%$ vs. $20 \%$ ( $p=0.007)$.

Estimating hemorrhagic risk for patients with ACS is not a simple task, since prognostic indexes present low accuracy, being even more complex in elderly patients. In the Spanish LONGEVO-SCA (Impacto de la fragiLidad y Otros síNdromes GEriátricos en el manejo y pronóstico Vital del ancianO con Síndrome Coronario Agudo sin elevación de segmento ST) registry, which included patients older than 80 , with $78 \%$ of the population being submitted to an invasive strategy, the rate of major bleeding was only $7 \%$. In the analysis of age-related variables such as comorbidities, physical disability, cognitive deficit, frailty and risk of malnutrition, only the Charlson comorbidities index 
was associated as an independent variable for major bleeding. When associated with the CRUSADE score (area under the ROC curve $=0.64$ ), this index modestly improved the predictive capacity $(\mathrm{AUC}=0.68){ }^{3}$

The use of the SHARE-FI (Survey of Health, Ageing and Retirement in Europe Frailty Index) frailty index in patients $>75$ years and admitted for SCA (STEMI = $36.5 \%$ ) showed that frailty was an independent predictor of the composite of death and reinfarction (adjusted HR $2.81 ; 95 \% \mathrm{CI}=1.16-6.78$ ) and overall mortality (HR: 3.07; $95 \% \mathrm{CI}=1.35-6.98) .{ }^{4}$ These patients were also older, with higher comorbidity, GRACE and CRUSADE scores.

A recent systematic review comparing the use of more potent P2Y12 inhibitors (ticagrelor and prasugrel) with clopidogrel in elderly and non-elderly patients with ACS, demonstrated a significant reduction in efficacy outcomes in non-elderly patients $(\mathrm{RR}=0.85 ; 95 \% \mathrm{CI}=0.79$ - 0.93) but only a tendency of reduction in elderly patients (RR $=0.95 ; 95 \% \mathrm{CI}=0.86-1.05)$, with similar risks of bleeding among elderly $(\mathrm{RR}=1.19 ; 95 \% \mathrm{CI}=0.95-1.49])$ and nonelderly patients $(\mathrm{RR}=1.16 ; 95 \% \mathrm{CI}=0.95-1.41] .{ }^{5}$ In order to minimize this elevated bleeding risk of older patients, the ELDERLY ACS-2 study compared conventional doses of clopidogrel (300 mg / $75 \mathrm{mg}$ ) with reduced maintenance doses of prasugrel $(60 \mathrm{mg} / 5 \mathrm{mg}$ ) in patients (mean age: 80 years) admitted with ACS and submitted to PCI. ${ }^{6}$ Discontinuation of the trial due to futility for efficacy precludes correct interpretation of the non-significant $48 \%$ reduction in the rate of relevant bleedings.

Regarding the timing of first administration of P2Y12 inhibitors, a recent meta-analysis including 9,649 STEMI patients submitted to PCI, early administration compared to delayed use was associated with a significant reduction in major cardiac adverse events, myocardial infarction, "bail-out" use of glycoprotein inhibitors and better coronary TIMI flow 2 / 3 prior to PCI, without increasing bleeding rates. ${ }^{7}$

Paradoxically, elderly patients present lower response to antiplatelet agents and higher rates of hemorrhagic complications, a fact only partially understood.

In the SENIOR-PLATELETS study involving patients with stable coronary artery disease and chronic use (> 14 days) of acetylsalicylic acid and thienopyridine (clopidogrel $75 \mathrm{mg}$, clopidogrel $150 \mathrm{mg}$ or prasugrel
$10 \mathrm{mg}$ ), the elderly group had a higher rate of reactivity compared to younger patients (OR: 2.58, 95\% CI: 1.76 $3.79, \mathrm{p}<0.0001)$. A similar trend, although attenuated and without statistical significance, was observed with clopidogrel $150 \mathrm{mg}$ and prasugrel $10 \mathrm{mg} .{ }^{8}$

Elevated platelet reactivity appears to be due to higher baseline level in the elderly, but also to lower response to the drug. With regard to hyperresponding patients, elderly patients were in a smaller number, which should attenuate the higher hemorrhagic risk of this subgroup, a fact not observed in clinical practice, denoting that platelet activity is not the only determinant, and suggesting relevant interferences from comorbidities.

Individualization of antiplatelet doses based on functional tests has been evaluated as a strategy to modulate hemorrhagic and ischemic risks, but it has not been routinely recommended, even for the elderly. In the ANTARCTIC (Assessment of a Normal versus Tailored dose of prasugrel after stenting in patients Aged > 75 years to Reduce the Composite of bleeding, stent Thrombosis and Ischemic Complications) trial involving a population of high risk, constituted by elderly patients with ACS and PCI, the evaluation of platelet function by VerifyNow/P2Y12 modified the initial dose of prasugrel in $45 \%$ of patients but did not improve clinical outcomes. ${ }^{9}$

In the TROPICAL-ACS (Testing Responsiveness To Platelet Inhibition On Chronic Antiplatelet Treatment For Acute Coronary Syndromes) study, a strategy of dose titration of prasugrel guided by platelet functional assessment, compared to standard dose, did not prove to be non-inferior in terms of net clinical benefit. In patients $\leq 70$ years, the combined clinical events were more favorable in the guided group, especially for the reduction of hemorrhagic complications, but the same did not occur in the subgroup of older patients. ${ }^{10}$

In conclusion, ideal dual antiplatelet therapy in elderly patients remains unclear and clinical trials are especially recommended to define the type of drug, loading and maintenance dosing and duration of therapy. The development of new tools for better ischemic and hemorrhagic risk stratification in the increasing population of elderly patients admitted for ACS is also urgently needed. 


\section{References}

1. Redfors B, Dworeck C, Haraldsson I, Angerås O, Odenstedt J, Ioanes D, et al. Pre treatment with P2Y12 receptor antagonists in ST-elevation myocardial infarction: a report from the Swedish Coronary Angiography and Angioplasty Registry. Eur Heart J. 2019;40(15):1202-10.

2. Soeiro AM, Casale G, Lopes MAA, Godoy LC, Bossa AS, Biselli B, et al. Is there safety in the use of clopidogrel loading dose in patients over 75 yaears of age with acute coronary syndrome. Int J Cardiovasc Sci. 2019;32(5):449-456.

3. Ariza-Solé A, Guerrero C, Formiga F, Aboal J, Abu-Assi E, Marín F, et al. Global Geriatric Assessment and In-Hospital Bleeding Risk in Elderly Patients with Acute Coronary Syndromes: Insights from the LONGEVOSCA Registry. Thromb Haemost. 2018;118(3):581-90.

4. Alonso Salinas GL, Sanmartin M, Pascual Izco M, Rincon LM, MartinAcuna A, Pastor Pueyo P, et al. The Role of Frailty in Acute Coronary Syndromes in the Elderly. Gerontology. 2018;64(5):422-9.

5. Tarantini G, Ueshima D, D'Amico G, Masiero G, Musumeci G, Stone $\mathrm{GW}$, et al. Efficacy and safety of potent platelet $\mathrm{P} 2 \mathrm{Y} 12$ receptor inhibitors in elderly versus nonelderly patients with acute coronary syndrome: A systematic review and meta-analysis. Am Heart J. 2018;195:78-85.

6. Savonitto S, Ferri LA, Piatti L, Grosseto D, Piovaccari G, Morici N, et al. Comparison of Reduced-Dose Prasugrel and Standard-Dose Clopidogrel in Elderly Patients With Acute Coronary Syndromes Undergoing Early Percutaneous Revascularization. Circulation. 2018;137(23):2435-45.

7. Bellemain-Appaix A, Bégué C, Bhatt DL, Ducci K, Harrington RA, Roe $\mathrm{M}$, et al. The efficacy of early versus delayed P2Y12 inhibition in percutaneous coronary intervention for ST-elevation myocardial infarction: a systematic review and meta-analysis. EuroIntervention. 2018;14(1):78-85

8. Silvain J, Cayla G, Hulot JS, Finzi J, Kerneis M, O'Connor SA, et al. High on-thienopyridine platelet reactivity in elderly coronary patients: the SENIOR-PLATELET study. Eur Heart J. 2012;33(10):1241-9.

9. Cayla G, Cuisset T, Silvain J, Leclercq F, Manzo-Silberman S, SaintEtienne $C$, et al. Platelet function monitoring to adjust antiplatelet therapy in elderly patients stented for an acute coronary syndrome (ANTARCTIC): an open-label, blinded-endpoint, randomised controlled superiority trial. Lancet. 2016;388(10055):2015-22.

10. Sibbing D, Gross L, Trenk D, Jacobshagen C, Geisler T, Hadamitzky M, et al. Age and outcomes following guided de-escalation of antiplatelet treatment in acute coronary syndrome patients undergoing percutaneous coronary intervention: results from the randomized TROPICAL-ACS trial. Eur Heart J. 2018;39(29):2749-58. 